\title{
ФУНКЦИИ БЕССЕЛЯ В ТЕОРИИ ОДНОМЕРНЫХ ВОЛНОВЫХ УРАВНЕНИИ
}

\section{Введение}

В предыдущей статье [ $\left.{ }^{1}\right]$ было рассмотрено несколько примеров одномерных волновых уравнений, получаемых из уравнения внда

$$
d^{2} U / d z^{2}+\left[G^{\prime 2}+G^{\prime \prime \prime} / 2 G^{\prime}-3 G^{\prime \prime 2} / 4 G^{\prime 2}-\left(v^{2}-1 / 4\right) G^{\prime 2} / G^{2}\right] U=0,
$$

где $U$ - полевая функция, $G(z)$ - произвольная функция, а $v-$ постоянная. Решение этого уравнения

$$
U=\left(G / G^{\prime}\right)^{1 / 2} Z_{v}(G)
$$

выражается через функцию Бесселя $v$-го порядка $Z_{v}$. Было найдено несколько таких форм функций $G(z)$, при которых

$$
G^{\prime 2}+G^{\prime \prime \prime} / 2 G^{\prime}-3 G^{\prime \prime 2} / 4 G^{\prime 2}-\left(v^{2}-1 / 4\right) G^{\prime 2} / G^{2}=k^{2} n^{2}(z),
$$

т. е. уравнение (1) получает вид

$$
d^{2} U / d z^{2}+k^{2} n^{2}(z) U=0 .
$$

Это и есть одномерное волновое уравнение, описывающее нормальное распространение монохроматического света с волновым числом $k$ в слоисто-неоднородной среде с показателем преломления $n(z)$. В настоящей статье найдем еще целую серию подобных уравнений, каждое из которых определяется некоторой функцией $G$ и имеет решение вида (2).

\section{Основное решение}

Нашим исходным уравнением является (3), в котором неизвестными функциями являются и $G(z)$, и $n(z)$. Будем искать $G(z)$ в виде

$$
G(z)=f^{p}(u(z)),
$$

где $p$ - безразмерная отличная от нуля постоянная. Вычисляя производные и подставляя их в уравнение (3), находим

$$
\begin{gathered}
{\left[p^{2} f^{2 p-2} f^{\prime 2}+f^{\prime \prime \prime} / 2 f^{\prime}-3 f^{\prime \prime 2} / 4 f^{\prime 2}-\left(v^{2} p^{2}-1 / 4\right) f^{\prime 2} / f^{2}\right] u^{\prime 2}+} \\
+u^{\prime \prime \prime} / 2 u^{\prime}-3 u^{\prime \prime 2} / 4 u^{\prime 2}=k^{2} n^{2},
\end{gathered}
$$

где штрих у $f$ означает производную по $u$, а у $u$ по $z$. Этому равенству можно удовлетворить, положив 


$$
\begin{gathered}
u^{\prime \prime \prime} / 2 u^{\prime}-3 u^{\prime \prime 2} / 4 u^{\prime 2}=0, \\
p^{2} f^{2 p-2} f^{\prime 2}+f^{\prime \prime \prime} / 2 f^{\prime}-3 f^{\prime \prime 2} / 4 f^{\prime 2}=0
\end{gathered}
$$

и

$$
1-4 v^{2} p^{2}=4 q^{2} k^{2} h^{2},
$$

где $q-$ произвольная безразмерная постоянная, а $h-$ толщина слоя. Последнее равенство определяет порядок функции Бесселя

$$
v=(2 p)^{-1}\left(1-4 q^{2} k^{2} h^{2}\right)^{1 / 2},
$$

а из уравнения (6) находим

$$
n^{2}=q^{2} h^{2} u^{\prime 2} f^{\prime 2} / f^{2} .
$$

Это выражение можно упростить. Так как

$$
u^{\prime} f^{\prime} / f=f^{-1} d f / d z=p^{-1} G^{-1} d G / d z,
$$

To

$$
n^{2}=q^{2} h^{2} p^{-2} G^{-2}(d G / d z)^{2} .
$$

Соответственно, решение (2) волнового уравнения получает вид

$$
U=n^{-1 / 2} Z_{v}(G) .
$$

Уравнения (7) и (8) определяют зависимость $u$ от $z$ и $f$ от $u$. Общее решение уравнения (7) таково

$$
u(z)=\frac{a_{1} z / h+a_{2}}{a_{3} z / h+a_{4}},
$$

где $a_{1}, a_{2}, a_{3}, a_{4}$ - безразмерные постоянные, удовлетворяющие условию

$$
a_{1} a_{4}-a_{2} a_{3}=1 .
$$

Уравнение (8) имеет, конечно, тривиальное решение $f=$ const, которое не представляет интереса. Опуская его, подстановкой

$$
f^{\prime}=y^{2}
$$

переводим уравнение в линейное уравнение второго порядка

$$
d^{2} y / d f^{2}+p^{2} f^{2 p-2} y=0 .
$$

Введем вместо $f$ в качестве аргумента $G=f^{p}$ и положим для удобства

$$
p=(2 m)^{-1}
$$

так что

$$
f=G^{2 m}
$$

Тогда

$$
d^{2} y / d G^{2}-(2 m-1) G^{-1} d y / d G+y=0 .
$$

Решением этого уравнения является

$$
y=G^{m} Z_{m}^{(1)}(G),
$$


где $Z_{m}{ }^{(1)}$ - одна из функций Бесселя $m$-го порядка. Выбор $Z_{m}^{(1)}$ произволен. Формулы (10) и (13) перепишем, согласно (19), в виде

$$
v=m\left(1-4 q^{2} k^{2} h^{2}\right)^{1 / 2}
$$

и

$$
n^{2}=4 q^{2} m^{2} h^{2} G^{-2}(d G / d z)^{2} .
$$

Далее найдем зависимость $u$ от $G$. Для этого имеем формулу (17), которую, согласно формулам (20) и (22), представим в виде

$$
d G / d u=(2 m)^{-1} G Z_{m}^{(1) 2}(G) .
$$

Отсюда

$$
d u=2 m G^{-1} Z_{m}^{(1)-2}(G) d G
$$

Интегрируя, находим

$$
u-u_{0}=2 m Z_{m}^{(2)}(G) / Z_{m}^{(1)}(G)
$$

где $Z_{m}{ }^{(2)}$ - другая, линейно независимая от $Z_{m}{ }^{(1)}$, функция Бесселя $m$-го порядка, причем обе выбраны так, что

$$
Z_{m}^{(1)} Z_{m}^{(2) \prime}-Z_{m}^{(1) \prime} Z_{m}^{(2)}=G^{-1}
$$

Упростим формулу (27) подстановкой $u \rightarrow 2 m u+u_{0}$; при одновременной подстановке $a_{1} \rightarrow(2 m)^{1 / 2} a_{1}+(2 m)^{-1 / 2} u_{0} a_{3}, \quad a_{2} \rightarrow(2 m)^{1 / 2} a_{2}+(2 m)^{-1 / 2} u_{0} a_{4}$, $a_{3} \rightarrow(2 m)^{-1 / 2} a_{3}, a_{4} \rightarrow(2 m)^{-1 / 2} a_{4}$ формулы $(15)$ и $(16)$ останутся неизменными, а формулы (25)-(27) примут вид

$$
\begin{gathered}
d G / d u=G Z_{m}^{(1) 2}(G), \\
d u=G^{-1} Z_{m}^{(1)-2}(G) d G
\end{gathered}
$$

и

$$
u=Z_{m}^{(2)}(G) / Z_{m}^{(1)}(G)
$$

Последняя формула определяет и зависимость между $z$ и $G$. Так как, согласно формуле (15),

$$
z=h \cdot \frac{a_{4} u-a_{2}}{a_{1}-a_{3} u},
$$

TO

$$
z=h \cdot \frac{a_{4} Z_{m}^{(2)}(G)-a_{2} Z_{m}^{(1)}(G)}{a_{1} Z_{m}^{(1)}(G)-a_{3} Z_{m}^{(2)}(G)}
$$

Теперь мы можем найти зависимость показателя преломления от $G$. Вычисляя производную $d u / d z$ и учитывая формулу (31), находим

$$
\begin{gathered}
d u / d z=h^{-1}\left(a_{3} z / h+a_{4}\right)^{-2}=h^{-1}\left(a_{1}-a_{3} u\right)^{2}= \\
=h^{-1} Z_{m}^{(1)-2}(G)\left[a_{1} Z_{m}^{(1)}(G)-a_{3} Z_{m}^{(2)}(G)\right]^{2} .
\end{gathered}
$$

Далее, учитывая формулу (29), имеем 


$$
d G / d z=(d G / d u)(d u / d z)=h^{-1} G\left[a_{1} Z_{m}^{(1)}(G)-a_{3} Z_{m}^{(2)}(G)\right]^{2} .
$$

Подставляя это выражение в формулу (24), находим

$$
n=2 q m\left[a_{1} Z_{m}^{(1)}(G)-a_{3} Z_{m}^{(2)}(G)\right]^{2} .
$$

Итак, мы нашли (формулы (33) и (36)) параметрическую зависимость показателя преломления от координаты. Параметром является $G$. Решение волнового уравнения

$$
d^{2} U / d z^{2}+4 k^{2} q^{2} m^{2}\left[a_{1} Z_{m}^{(1)}(G)-a_{3} Z_{m}^{(2)}(G)\right]^{4} U=0
$$

с этим показателем преломления выражается формулой (14), которую напишем, согласно формулам (23) и (36), в виде (опуская несущественный постоянный множитель)

$$
U=\left[a_{1} Z_{m}^{(1)}(G)-a_{3} Z_{m}^{(2)}(G)\right]^{-1} Z_{m\left(1-4 q^{2} k^{2} h^{2}\right)^{1 / 2}}(G) .
$$

Исключение параметра возможно в единственном случае, именно при $m=1 / 2$. Однако, как было показано в $\left[{ }^{2}\right]$, параметрическая форма зависимости показателя преломления и решения волнового уравнения от координаты не препятствует нахождению всех могущих интересовать нас оптических характеристик неоднородной пленки.

\section{Частные случаи}

Особо следует рассмотреть случай $m=0$. Он не входит непосредственно в полученный выше общий результат, так как $m=0$ означает $p \rightarrow \infty$ и $n=0$. Однако мы можем принять одновременно с $m=0$ $q \rightarrow \infty$. Пусть

$$
\lim _{m \rightarrow 0}(m q)=Q .
$$

Тогда показатель преломления равен

$$
n=2 Q\left[a_{1} Z_{0}^{(1)}(G)-a_{3} Z_{0}^{(2)}(G)\right]^{2},
$$

волновое уравнение имеет вид

$$
d^{2} U / d z^{2}+4 k^{2} Q^{2}\left[a_{1} Z_{0}^{(1)}(G)-a_{3} Z_{0}^{(2)}(G)\right]^{4} U=0,
$$

решение его есть

$$
U=\left[a_{1} Z_{0}^{(1)}(G)-a_{3} Z_{0}^{(2)}(G)\right]^{-1} Z_{2 i Q k h}(G),
$$

а формула (33) переходит в

$$
z=h \cdot \frac{a_{4} Z_{0}^{(2)}(G)-a_{2} Z_{0}^{(1)}(G)}{a_{1} Z_{0}^{(1)}(G)-a_{3} Z_{0}^{(2)}(G)} .
$$

Рассмотрим далее случай $m=1 / 2$, допускающий исключение параметра $G$. Выберем $Z_{1 / 2}{ }^{(1)}(G)$ и $Z_{1 / 2}{ }^{(2)}(G)$ в виде

$$
\begin{aligned}
& Z_{1 / 2}^{(1)}=G^{-1 / 2} \cos (G+K) \\
& Z_{1 / 2}^{(2)}=G^{-1 / 2} \sin (G+K)
\end{aligned}
$$


где $K$ - произвольная постоянная. Тогда, согласно формуле (33),

$$
G=\arctan \left(\frac{a_{1} z / h+a_{2}}{a_{3} z / h+a_{4}}\right)-K .
$$

Подставляя это выражение в формулы (36)-(38) и учитывая формулы (16) и (44), получаем формулу показателя преломления

$$
\begin{gathered}
n(z)=q\left[\left(a_{1}^{2}+a_{3}^{2}\right) z^{2} / h^{2}+2\left(a_{1} a_{2}+a_{3} a_{4}\right) z / h+\left(a_{2}^{2}+a_{4}^{2}\right)\right]^{-1} \times \\
\times\left[\arctan \left(\frac{a_{1} z / h+a_{2}}{a_{3} z / h+a_{4}}\right)-K\right]^{-1},
\end{gathered}
$$

волновое уравнение

$$
\begin{gathered}
d^{2} U / d z^{2}+k^{2} q^{2}\left[\left(a_{1}^{2}+a_{3}^{2}\right) z^{2} / h^{2}+2\left(a_{1} a_{2}+a_{3} a_{4}\right) z / h+\left(a_{2}^{2}+a_{4}^{2}\right)\right]^{-2} \times \\
\times\left[\arctan \left(\frac{a_{1} z / h+a_{2}}{a_{3} z / h+a_{4}}\right)-K\right]^{-2} U=0
\end{gathered}
$$

и его решение

$$
\begin{gathered}
U(z)=\left[\left(a_{1}^{2}+a_{3}^{2}\right) z^{2} / h^{2}+2\left(a_{1} a_{2}+a_{3} a_{4}\right) z / h+\left(a_{2}^{2}+a_{4}^{2}\right)\right]^{1 / 2} \times \\
\times\left[\arctan \left(\frac{a_{1} z / h+a_{2}}{a_{3} z / h+a_{4}}\right)-K\right]^{1 / 2} Z_{\left(1 / 4-q^{2} h^{2} h^{2}\right)^{1 / 2}}\left(\arctan \left(\frac{a_{1} z / h+a_{2}}{a_{3} z / h+a_{4}}\right)-K\right) .
\end{gathered}
$$

Целесообразно рассмотреть отдельно еще случай произвольного полуцелого значения $m$, когда функции $Z_{m}$ выражаются через элементарные функции. Обозначим

$$
m=\mu+1 / 2,
$$

где $\mu$ - неотрицательное целое число. Функциям $Z^{(1)} \mu+1 / 2$ и $Z^{(2)} \mu+1 / 2$ дадим следующее выражение

$$
Z_{\mu+1 / 2}^{(1)}(G)=G^{-\mu-1 / 2}\left[A_{\mu}(G) \cos (G+K)-B_{\mu}(G) \sin (G+K)\right]
$$

и

$$
Z_{\mu+1 / 2}^{(2)}(G)=G^{-\mu-1 / 2}\left[A_{\mu}(G) \sin (G+K)+B_{\mu}(G) \cos (G+K)\right],
$$

где $K$ - произвольная постоянная, а $A_{\mu}(G)$ и $B_{\mu}(G)-$ полиномы, определяемые формулами

$$
A_{\mu}(G)=\sum_{l=0}^{\infty} \frac{(-1)^{l}(\mu+2 l) ! G^{\mu-2 l}}{2^{2 l}(2 l) !(\mu-2 l) !}
$$

и

$$
B_{\mu}(G)=\sum_{l=0}^{\infty} \frac{(-1)^{l}(\mu+2 l+1) ! G^{\mu-2 l-1}}{2^{2 l+1}(2 l+1) !(\mu-2 l-1) !}
$$

(суммирование до бесконечности показано формально; на самом деле суммы при достаточно большом $l$ обрываются). Функции $Z^{(1)}{ }^{\mu+1 / 2}$ и $Z^{(2)}{ }_{\mu+1 / 2}$ удовлетворяют, как легко убедиться, соотношению (28). Для $z$, 
$n$, волнового уравнения и его решения находйм следующие формулы:

$$
\begin{gathered}
z=h \cdot \frac{\left[a_{4} A_{\mu}(G)+a_{2} B_{\mu}(G)\right] \tan (G+K)-\left[a_{2} A_{\mu}(G)-a_{4} B_{\mu}(G)\right]}{\left[a_{1} A_{\mu}(G)-a_{3} B_{\mu}(G)\right]-\left[a_{3} A_{\mu}(G)+a_{1} B_{\mu}(G)\right] \tan (G+K)}, \\
n=(2 \mu+1) q G^{-2 \mu-1}\left\{\left[a_{1} A_{\mu}(G)-a_{3} B_{\mu}(G)\right] \cos (G+K)-\right. \\
\left.-\left[a_{3} A_{\mu}(G)+a_{1} B_{\mu}(G)\right] \sin (G+K)\right\}^{2}, \\
d^{2} U / d z^{2}+k^{2} q^{2}(2 \mu+1)^{2} G-4 \mu-2\left\{\left[a_{1} A_{\mu}(G)-\right.\right. \\
\left.\left.-a_{3} B_{\mu}(G)\right] \cos (G+K)-\left[a_{3} A_{\mu}(G)+a_{1} B_{\mu}(G)\right] \sin (G+K)\right\}^{4} U=0
\end{gathered}
$$

Н

$$
\begin{array}{r}
U=G^{\mu+1 / 2}\left\{\left[a_{1} A_{\mu}(G)-a_{3} B_{\mu}(G)\right] \cos (G+K)-\right. \\
\left.-\left[a_{3} A_{\mu}(G)+a_{1} B_{\mu}(G)\right] \sin (G+K)\right\}^{-1} Z_{(2 \mu+1)\left(1 / 4-q^{2} h^{2} h^{2}\right)^{1 / 2}}
\end{array}
$$

В случае $\mu=0$ из этих формул по исключении $G$ вытекают формулы (46) $-(48)$.

\section{Л Н Т Е А Т Р А}

1. К а р д П., Изв. АН ЭССР, Физ. Матем., 29, № 1, 1-7 (1980).

2. Ка р д П., Изв. АН ЭССР, Физ. Матем., 26, № 3, 252-259. (1977).

Тартуский государственный университет
Поступила в редакщию $3 / \mathrm{X} 1979$

\section{P. KARD}

\section{BESSELI FUNKTSIOONID OHEMŌOTMELISTE LAINEVORRANDITE TEOORIAS}

Uhemõõtmeline lainevõrrand (4), kus $U$ on väljasuurus, $z$ koordinaat, $n(z)$ murdumisnäitaja ja $k$ lainearv, kirjeldab elektromagnetlaine levimist mittehomogeenses kihilises keskkonnas normaalse langemise juhul. Vörrandi (3) kehtivuse korral, kus $G$ on mingi funktsioon $z$-st, avaldub lainevōrrandi lahend valemiga (2), kus $Z_{v}$ on $v$-ndat järku Besseli funktsioon. Võrrandit (3) rahuldavate $G(z)$ ja $n(z)$ leidmiseks eeldatakse seoste (5), (7), (8) ja (10) kehtivust, kus $p$ ja $q$ on dimensioonitud nullist erinevad konstandid ja $h$ on kihi paksus. Siis kehtib ka avaldis (13). Vōrrandi (7) üldlahend on (15), kus $a_{1}, a_{2}, a_{3}, a_{4}$ on dimensioonitud konstandid, mis rahuldavad tingimust (16). Võrrandile (8) leitakse lahend kujul (31), kus $m=(2 p)^{-1}$, kuna $Z_{m}{ }^{(1)}$ ja $Z_{m}^{(2)}$ on $m$-ndat järku lineaarselt sõltumatud Besseli funktsioonid vronskiaaniga $G^{-1}$. Valemitest (13), (15) ja (31) järgnevad valemid (33) ja (36), mis määravad murdumisnäitaja sõltuvuse koordinaadist parameetri $G$ kaudu. Lainevōrrand ja ta lahend on antud valemites (37) ja (38). Piirjuhul $m=0$ kehtivad valemid $(40)-(43)$. Parameetri elimineerimine on võimalik vaid juhul $m=1 / 2$, mispuhul kehtivad valemid (45)-(48). Kui $m=\mu+1 / 2$ ning $\mu$ on mittenegatiivne täisarv, siis kehtivad valemid $(54)-(57)$, kus $A_{\mu}(G)$ ja $B_{\mu}(G)$ on valemitega (52) ja (53) defineeritud polünoomid. 


\section{P. KARD}

\section{BESSEL FUNCTIONS IN THE THEORY OF THE ONE-DIMENSIONAL WAVE EQUATIONS}

When $U$ denotes the field quantity, $z$ the co-ordinate, $n(z)$ the refractive index and $k$ the wave number, then the equation (4) has the meaning of the wave equation, describing the propagation of the electromagnetic wave through an inhomogeneous layered medium in the case of the normal incidence. If the formula (3) holds, where $G$ is a function of the co-ordinate, then the solution of the wave equation is of the form (2), where $Z_{v}$ is Bessel function of the $v$-th order. To find $n(z)$ and $G(z)$ satisfying the equation (3), the relations (5), (7), (8), and (10) are assumed, where $p$ and $q$ are dimensionless non-zero constants and $h$ is the thickness of the layer. Then formula (13) holds too. The general solution of the equation (7) is of the form (15), where $a_{1}, a_{2}, a_{3}, a_{4}$ are dimensionless constants, satisfying the equality (16). The solution of the equation $(8)$ is done by the formula $(31)$, where $m=(2 p)^{-1}$ and $Z_{m}{ }^{(1)}, Z_{m}{ }^{(2)}$ are linearly independent Bessel functions of the $m$-th order with the wronskian equal to $G^{-1}$. From the formulae (13), (15), and (31) follow formulae (33) and (36), by which the dependence of the refractive index upon the co-ordinate via parameter $G$ is established. The wave equation and its solution are presented by the formulae (37) and (38). In the limiting case $m=0$ the formulae $(40)-(43)$ hold. One cannot eliminate the parameter unless $m=1 / 2$; in this case formulae (45) - (48) hold. When $m=\mu+1 / 2$, $\mu$ being a non-negative integer, the formulae $(54)-(57)$ hold, where $A_{\mu}(G)$ and $B_{\mu}(G)$ are polynomials defined by the formulae (52) and (53). 